\title{
Dynamics of anticipatory mechanisms during predictive context processing
}

\author{
Aurélie Bidet-Caulet ${ }^{a}$, Pierre-Guillaume Barbe $^{b, c}$, Sylvie Roux ${ }^{c, d}$, Humsini Viswanath ${ }^{a}$, \\ Catherine Barthélémy ${ }^{\mathrm{c}, \mathrm{d}, \mathrm{e}}$, Nicole Bruneau ${ }^{\mathrm{d}}$, Robert T. Knight ${ }^{\mathrm{a}, \mathrm{f}}$, and Frédérique Bonnet- \\ Brilhault ${ }^{\mathrm{a}, \mathrm{c}, \mathrm{d}, \mathrm{e}}$ \\ aHelen Wills Neuroscience Institute, University of California, Berkeley, 132 Barker Hall, Berkeley, \\ CA 94720-3190, USA

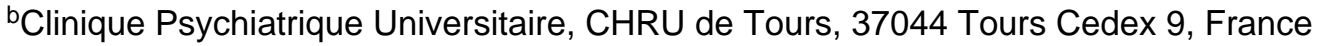 \\ 'Université François Rabelais, Tours, France \\ 'INSERM, UMR930, CHRU Bretonneau, 2 boulevard Tonnellé, 37044 Tours Cedex, France \\ eCentre Universitaire de Pédopsychiatrie, CHRU de Tours, 2 boulevard Tonnellé 37000 Tours, \\ France \\ fDepartment of Psychology, University of California, Berkeley, Tolman Hall University of \\ California, Berkeley, CA 94720-1650, USA
}

\begin{abstract}
We employed an EEG paradigm manipulating predictive context to dissociate the neural dynamics of anticipatory mechanisms. Subjects either detected random targets or targets preceded by a predictive sequence of three distinct stimuli. The last stimulus in the 3-stimulus sequence (decisive stimulus) did not require any motor response but $100 \%$ predicted a subsequent target event. We show that predictive context optimizes target processing via the deployment of distinct anticipatory mechanisms at different times of the predictive sequence. Prior to the occurrence of the decisive stimulus, enhanced attentional preparation was manifested by reductions in the alpha oscillatory activities over visual cortices, resulting in facilitation of processing of the decisive stimulus. Conversely, the subsequent $100 \%$ predictable target event did not reveal deployment of attentional preparation in the visual cortices, but elicited enhanced motor preparation mechanisms, indexed by an increased contingent negative variation (CNV) and reduced mu oscillatory activities over motor cortices before movement onset. The present results provide evidence that anticipation operates via different attentional and motor preparation mechanisms by selectively pre-activating task-dependent brain areas as predictability gradually increases.
\end{abstract}

\section{Keywords}

attention; motor preparation; alpha; mu; beta

\section{Introduction}

Extraction of informative cues from our surrounding environment allows us to predict and anticipate upcoming events and to guide our behavior. Thus, a predictive context can be used to facilitate the selection of an appropriate response via the deployment of several

Corresponding author: Aurélie Bidet-Caulet, Inserm Unité U1028, 69675 Bron Cedex, France, Phone \#: +33 (0) 472 13 89 31, Fax \#: +33(0) 4721389 01, aurelie.bidet-caulet@inserm.fr. 
anticipatory/preparatory mechanisms. Indeed, anticipation of upcoming predictable events facilitates the processing of the upcoming stimulus, and improves the selection and execution of the behavioral response.

In the attention domain, both scalp EEG and fMRI studies show that anticipatory attention increases the processing of expected stimuli (see Hillyard et al., 1998, for a review), resulting in facilitated stimulus detection (Posner, 1980; Fogelson et al., 2009; Fogelson et al., 2010b). These attention effects support the deployment of preparatory processes, i.e. attentional preparation, during stimulus expectancy. Using fMRI, enhanced preparatory activity is found in visual (Kastner et al., 1999) and auditory (Voisin et al., 2006) areas. This preparatory biasing is also observed from EEG recordings as a decrease in the alpha frequency band (around $10 \mathrm{~Hz}$ ) in visual areas contra-lateral to the attended stimulus (Worden et al., 2000; Sauseng et al., 2005; Thut et al., 2006; Rihs et al., 2007; Trenner et al., 2008; Kelly et al., 2009), and as an alpha increase in ipsi-lateral visual areas (Worden et al., 2000; Rihs et al., 2007). Therefore, alpha activity seems to reflect the active inhibition of brain areas not involved in the current brain operations, and a decrease in alpha power would correspond to a release of inhibition and an enhanced excitability of the cortical regions processing the expected visual stimuli (see Klimesch et al., 2007, for reviews; Jensen \& Mazaheri, 2010).

In the motor domain, preparation of a movement is associated with reductions (also called desynchronization) in the mu (around $10 \mathrm{~Hz}$ ) and the beta (around $20 \mathrm{~Hz}$ ) rhythms, which are typically observed at central electrodes and are followed by a transient increase in beta power (see Pfurtscheller \& Lopes da Silva, 1999, for reviews; Hari, 2006). These mu and beta power reductions are proposed to index pre-activation of motor areas and to reflect motor preparation mechanisms.

Finally, several scalp EEG studies report sustained fronto-central maximal contingent negative variation (CNV) event-related potentials (ERPs) reflecting both attentional and motor preparation for the imperative stimulus (see Brunia \& van Boxtel, 2001, for a review).

This research reveals that anticipation relies on several distinct preparatory mechanisms than can be measured with scalp EEG. The aim of the current study was to investigate, in both the time and frequency domains, the dynamics of the different anticipatory mechanisms in predictive vs. non-predictive contexts. We focused our work on a rarely addressed methodological issue, namely the distinction and relationships between (stimulus phaselocked) event-related potentials and (non phase-locked) induced oscillatory activities. Using a sequence of predictive contextual cues (adopted from Fogelson et al., 2009), we were able to identify and dissociate several brain responses and examine their functional significance in contextual cue encoding, predictive information extraction, and anticipation.

\section{Materials and Methods}

\subsection{Subjects}

Fifteen right-handed volunteers ( 7 men; mean age $=26,1 \pm 1,2$ years) participated in this experiment. Ten were recruited at the University of Tours, and five at the University of California, Berkeley. All subjects were right-handed and had normal or corrected to normal vision. Inclusion criteria were no history of psychiatric disorders, no history of neurological disorder, and no substance abuse or dependence within the 6 months before the start of the study. Each participant gave written informed consent. This protocol was approved by the Committee for the Protection of Human Subjects for University California, Berkeley, and all subjects gave written informed consent. 


\subsection{Stimuli and Tasks}

Subjects sat in a chair in a sound-attenuated room, $110 \mathrm{~cm}$ in-front of a 21-inch PCcomputer screen. The experimenters and computers delivering visual stimuli and recording the EEG were located in a separate room. We used a paradigm designed to investigate visual context processing (adopted from Fogelson et al., 2009). Stimuli were presented centrally on a computer screen and subtended $3^{\circ}$ of visual angle (Fig. 1). Stimuli consisted of $15 \%$ targets (downward facing triangle) and $85 \%$ of equal amounts of three types of standards: triangles facing left, upward, or right. A target could be a random target (randT) preceded by a non-informative context (random sequence of stimuli) or a predicted target (predT) preceded by an informative context, i.e. a 3-stimuli predictive sequence (leftward, upward and rightward facing triangles). Triangles of the predictive sequence are labeled as predS1, predS2 and predS3 stimuli, whereas the corresponding triangles outside of the predictive sequence are labeled as randS1, randS2 and randS3, for leftward, upward and rightward facing triangles, respectively. We refer to the predS3 stimulus as the decisive stimulus. In each block (approximately $2.3 \mathrm{~min}$ long), a total of 127 stimuli (11 randT, 28 randS1, 28 randS3, 28 randS2, 8 predT, 8 predS1, 8 predS2, and 8 predS3) were presented each for 150 msec with an ISI of 1s. Each session consisted of 15 blocks, leading to a total of 165 randT, 420 randS1, 420 randS3, 420 randS2, 120 predT, 120 predS1, 120 pred2, and 120 predS3, for each participant.

Subjects performed a brief training session to ensure they were able to detect the target accurately. Subjects were then introduced to the predictive sequence before the recordings began and were aware that it would be $100 \%$ predictive of a target, but that targets would also appear randomly throughout the block. Participants were instructed to press a button with the right-hand index finger in response to target stimuli (downward facing triangles) and to look for the predictive sequence. Subjects were asked to centrally fixate throughout the recording. Stimulus presentation and response recordings were controlled using the Presentation software (Neurobehavioral Systems, Albany, CA, USA).

\subsection{EEG recording}

The EEG was recorded from 64 electrodes using identical ActiveTwo systems in Berkeley and Tours (Biosemi, The Netherlands). Vertical eye movements were monitored using electrodes placed above and below the left eye. The signal was recorded with a sampling frequency of $512 \mathrm{~Hz}$ and filtered at 0-104 Hz. Data were referenced offline to the tip of the nose.

\subsection{EEG data analysis}

EEG analyses were performed on standard and target visual stimuli embedded or not in the predictive sequence. We excluded from further analysis trials corresponding to standards after a target, standards before or after a button press, an randS2 standard preceded by a randS1 standard but not followed by a randS3 standard (since it is a potential predS2 standard), missed targets, and targets preceded by less than 3 standards. Trials contaminated with eye movements, eye blinks or excessive muscular activity in the [-700; $700 \mathrm{msec}]$ timewindow relative to stimulus onset were also excluded. In three subjects, an excessive number of trials were contaminated by a blink-related ocular activity. Independent Component Analysis (ICA) is a technique which provides a spatial filter that captures these blink artifacts in a limited number of ICs (1 or 2). Once identified, these ICs were selectively removed during the inverse ICA transformation to clean the data. In six subjects, the flat or excessively noisy signals at one or two electrodes were replaced by their values interpolated from the remaining electrodes using spherical spline interpolation (Perrin et al., 1989). 
Since the number of trials for stimuli embedded in the predictive sequence was lower than for the other stimuli, we equalized the number of trials within each pair of to-be-compared stimuli by random selection, for each participant. On average across participants, we obtained $100 \pm 13,98 \pm 14,86 \pm 11$, and $99 \pm 11$ trials for randS1/predS1, randS2/predS2, randS3/predS3 and randT/predT pairs, respectively, for each participant.

\subsection{Event-related potential analysis}

We averaged single trials, locked to stimulus onset, separately for each of the 8 stimulus categories (randS1, randS3, randS2, randT, predS1, predS2, predS3, predT). The resulting event-related potentials (ERPs) were digitally band-pass filtered between .5 and $30 \mathrm{~Hz}$ to analyze slower components, or between 4 and $30 \mathrm{~Hz}$ to extract early and fast sensory responses by filtering out slow and large components (such as CNV and P3) that can overlap fast and small responses (see SupFig. 1). For post-stimulus analysis, ERPs were corrected with a -100 to $0 \mathrm{msec}$ baseline before stimulus onset. For pre-stimulus analysis, ERPs were not baseline corrected. ERP scalp topographies were computed using spherical spline interpolation (Perrin et al., 1987; Perrin et al., 1989).

\subsection{Time-Frequency analysis}

We analyzed oscillatory activities by means of a wavelet decomposition which provides a good compromise between time and frequency resolutions. Each single trial signal was transformed in the time-frequency (TF) domain by convolution with complex Gaussian Morlet's wavelets with a ratio $f / \sigma f$ of 7 with $f$ being the central frequency of the wavelet and $\sigma f$ its standard deviation (see Tallon-Baudry \& Bertrand, 1999, for details). This leads to a wavelet duration of $202 \mathrm{msec}$ or $101 \mathrm{msec}$, and to a spectral bandwidth of $3.1 \mathrm{~Hz}$ or $6.3 \mathrm{~Hz}$ at $11 \mathrm{~Hz}$ or $22 \mathrm{~Hz}$, respectively. Thus, the time resolution of this method increases with frequency whereas the frequency resolution decreases. The resulting TF powers were then averaged across trials. This method led to a power estimate of both evoked (phase-locked to stimulus onset) and induced (jittering in latency) activities in the TF domain.

To assess the deployment of oscillatory activities throughout the 4-stimulus predictive sequence, we analyzed the oscillation power on a large time window [-500; $500 \mathrm{msec}]$ around each stimulus onset, and we applied the same baseline correction to all stimuli by subtracting the mean power between -500 and -250 msec before randS1 (embedded or not in the predictive sequence) onset (i.e. potential onset of a predictive sequence), in each frequency band.

To distinguish induced oscillatory activities from phase-locked evoked activities (reflecting the frequency content of ERPs), we computed, at each point of the TF domain, the stimulus phase-locking factor (PLF) from the single-trial TF analysis (Tallon-Baudry et al., 1996). This factor ranges from 0 (uniform phase distribution, i.e., high latency jitter) to 1 (strict phase-locking to the stimulus). The Rayleigh statistic was used to test for the non-uniformity of phase distribution (Jervis et al., 1983). When the PLF was less than .17, oscillations were considered to be non phase-locked to the stimulus.

We focused our analysis on 2 well-documented frequency bands: alpha or mu $(8-14 \mathrm{~Hz})$ and beta $(14-30 \mathrm{~Hz})$.

\subsection{Statistical Analysis}

To limit assumption on the data distribution, we used a statistical test based on randomizations (Edgington, 1995). Each randomization consisted in (1) the random permutation of the 15 pairs (corresponding to the 15 subjects) of values, (2) the sum of squared sums of values in the 2 obtained samples, and (3) the computation of the difference 
between these two statistic values. We did $2^{\wedge} 15=32768$ (maximum number of possible permutations) such randomization to obtain an estimate of the distribution of this difference under the null hypothesis. We then compared the actual difference between the values in the 2 conditions of interest to this distribution.

For statistical analysis of EEG data and to limit assumptions on the location and latency of the effects, we performed randomization tests for each of the 64 electrodes on several successive time-windows, and corrected for multiples tests. In the temporal dimension, we used a randomization procedure (Blair \& Karniski, 1993) to estimate the minimum number of consecutive time-windows that must be significant for the effect to be globally significant in the entire time-window of interest. For the spatial dimension, we considered as significant an effect visible at 4 or more adjacent electrodes.

The results of the randomization tests are illustrated on topographical views at a typical latency (usually at the maximum of the difference between conditions). As examples, corresponding ERP time-courses or time-frequency plots are depicted for a typical electrode showing a significant effect.

2.7.1 Statistical Analysis of Behavioral data-A button press within the interval of $100-1100 \mathrm{msec}$ after a target onset was considered a correct response, and a press at any other time was counted as a false alarm. Reaction times and accuracy were averaged for random and predicted targets, separately. The effect of predictability on these measures was assessed using a permutation test (see above).

2.7.2 Statistical Analysis of ERPs and oscillatory activities-To investigate the effect of predictability on both ERPs and oscillatory activities, we compared responses to the same physical stimuli embedded (predictive stimuli) or not (non-predictive) in the predictive sequence, i.e. we compared predS2 with randS2, predS3 with randS3, and predT with randT. No difference was predicted and none was observed between predS1 and randS1 since participants did not know at that time if the stimulus was part of the predictive sequence or not.

To analyze early and fast ERPs, we computed the permutation test on the 4-30Hz band-pass filtered ERP (pre-stimulus baseline corrected) amplitude at every sample of the [0;350 msec] time-window relative to stimulus onset. To analyze post-stimulus slow ERPs, we computed the permutation test on the $.5-30 \mathrm{~Hz}$ band-pass filtered ERP (pre-stimulus baseline corrected) mean amplitude in successive 20-msec time-windows of the [200; $600 \mathrm{msec}]$ total analysis window. To analyze pre-stimulus slow ERPs, we computed the permutation test on the $.5-30 \mathrm{~Hz}$ band-pass filtered ERP (not baseline corrected) mean amplitude in successive 20-msec time-windows of the [-500; $0 \mathrm{msec}]$ total analysis window.

For oscillatory activities, to reduce the effect of intersubject variability in frequency and latency, as well as the number of statistical comparisons, the permutation test was applied to mean TF energy values within smoothing TF windows regularly shifted by $50 \mathrm{msec}$ to cover the entire analysis time window $[-500 ; 500 \mathrm{msec}]$. According to the principle of wavelet analysis, the size of the smoothing TF windows differed with the frequency band of interest, varying from long duration and narrow bandwidth in the alpha range to shorter duration and larger bandwidth in the beta range. We used $200 \mathrm{msec} \times 6 \mathrm{~Hz}(8-14 \mathrm{~Hz})$ and $100 \mathrm{msec} \times 16$ $\mathrm{Hz}(14-30 \mathrm{~Hz}) \mathrm{TF}-$ windows to compare mean power of alpha and beta band oscillations, respectively. 
2.7.3 Specific statistical Analysis of the P3 ERP to targets-For the P3 ERP to targets, we extracted the latency and amplitude of the P3 maximum peak in the [300; 550 msec] time-window, and computed the permutation test on these values.

All signal and statistics analyses were performed with ELAN-Pack software developed at the Lyon Neuroscience Research Center, in France (Aguera et al., 2011) (http:// elan.lyon.inserm.fr/).

\section{Results}

\subsection{Behavioral results}

Participants performed the task well with mean accuracies of $99.0 \pm 1.2 \%$ and $99.4 \pm .7 \%$ to random and predicted targets, respectively, and few false alarms $(2.9 \pm 1.7)$. The reaction times (RT) to the predicted targets (mean RT $=376 \pm 47 \mathrm{msec}$ ) were shorter than those to the random targets (mean RT $=458 \pm 62 \mathrm{msec} ; \mathrm{p}<.0001$ ).

\subsection{ERP results}

3.2.1 predS2 stimulus-The only significant difference between the predS2 predictive stimulus and its non-predictive analog (randS2) was found between 380 and $460 \mathrm{msec}$ at multiple fronto-central electrodes $(\mathrm{p}<.01)$. This effect corresponds to an enhancement of the P3 ERP to standard stimuli when the stimulus had a predictive value (Fig. 2).

3.2.2 predS3 decisive stimulus-Early visual ERPs to the predS3 predictive and decisive stimulus, in comparison to its non-predictive analog (randS3), were significantly enhanced between 130 and $160 \mathrm{msec}$ (N1 latency) and between 190 and $220 \mathrm{msec}$ (P2 latency) at occipital electrodes ( $<<.01$; Fig. 3). The P3 ERP to the predS3 predictive stimulus was also enhanced between 280 and $500 \mathrm{msec}$ at parieto-central electrodes ( $\mathrm{p}<$. 001; Fig. 2).

3.2.3 predicted target-The CNV ERP before the predicted target (predT) significantly increased in comparison to the random target (randT), between -150 and $0 \mathrm{msec}$ before target onset, at central electrodes ( $\mathrm{p}<.01$; Fig. 4). The fronto-central components of the P2 and N2 ERPs observed in response to targets were reduced to the predicted target, between 200 and $250 \mathrm{msec}$ and between 290 and $330 \mathrm{msec}$, respectively, at fronto-central electrodes $(\mathrm{p}<.01 ;$ Fig. 5). The P2 amplitude was also significantly reduced at occipital electrodes, but no significant effect was found on the early visual ERPs before $200 \mathrm{~ms}$. The P3 ERP to the predicted target was earlier in latency $(\mathrm{p}<.01)$ and larger in amplitude $(\mathrm{p}<.05)$ in response to the predicted target (Fig. 2).

\subsection{Time-Frequency results}

Time-frequency analysis isolated two oscillatory phenomena in response to all stimuli, one at occipital electrodes indexing visual processing; and one at left central electrodes reflecting the activation of the motor cortex contra-lateral to the hand pressing the button.

At occipital electrodes (see Fig. 6 for randS3) an increase in alpha power was observed before stimulus onset, followed by a decrease in alpha power peaking around $400 \mathrm{msec}$ after stimulus onset. At the same time, a decrease in beta power, initiated before stimulus onset and peaking around $200 \mathrm{msec}$ after stimulus onset, was also prominent at occipital electrodes. Analysis of the phase-locking factor showed that increase in phase-locking is only visible after stimulus onset in the alpha and beta bands, superimposed to power decreases in the same frequency bands. This increase in phase-locking corresponds to the alpha and beta content of the early visual ERPs at these same electrodes. 
At left central electrodes a decrease in beta power was initiated before stimulus onset and peak around $200 \mathrm{msec}$ after stimulus onset (see Fig. 7 for randS3). Moreover, in response to targets, the decrease in beta power was followed by a decrease in mu power around motor execution, and a beta increase (or rebound) after the movement (see Fig. 7 for randT). Analysis of the phase-locking factor showed that increase in phase-locking is only visible after stimulus onset in the alpha band, superimposed to power decreases in the same frequency band. This increase in phase-locking corresponds to the alpha content of the fronto-central ERPs at these same electrodes.

Increases in alpha or beta power were not accompanied by simultaneous increases in phaselocking, indicating that the observed oscillatory phenomena are rather induced and nonphase-locked to the stimulus.

3.3.1 predS2 stimulus-We did not find any significant difference in oscillatory activities between the predS2 predictive stimulus and its non-predictive analog.

3.3.2 predS3 decisive stimulus-Beta power was significantly reduced to the predS3 predictive stimulus between $200 \mathrm{msec}$ pre-stimulus and stimulus onset at parieto-occipital electrodes ( $<$.01; Fig. 6). Alpha power was reduced to the predS3 predictive stimulus between $350 \mathrm{msec}$ pre-stimulus and $450 \mathrm{msec}$ post-stimulus at left parieto-occipital electrodes $(\mathrm{p}<.01$; Fig. 6$)$. This alpha effect corresponded to a reduced increase in alpha power before the predS3 stimulus in comparison to its non-predictive analog. These occipital effects were followed by a larger beta decrease to the predS 3 stimulus between 200 and $400 \mathrm{msec}$ at left central electrodes ( $<.01$; Fig. 7).

3.3.3 predicted target-Mu power was significantly reduced to the predicted target between $50 \mathrm{msec}$ pre-stimulus and $500 \mathrm{msec}$ post-stimulus at left central electrodes $(\mathrm{p}<$. 001; Fig. 7). Noteworthy, we did not observe any significant pre-stimulus alpha decrease at occipital electrodes before the predicted target. No significant effect was observed in the beta band.

\section{Discussion}

We compared the brain activities generated in response to the same physical stimuli embedded in a predictive or a non-predictive sequence of contextual cues. Throughout the predictive sequence, contextual cues were successively encoded in order to extract predictive information and to facilitate the recruitment of anticipatory/preparatory mechanisms at different times. First, after the two earliest stimuli of the sequence, the participants anticipated the potential occurrence of a decisive cue (predS3) that fully predicted a subsequent target, but did not require a motor response. Second, after seeing all three stimuli of the predictive sequence (i.e. after the decisive stimulus), the participants could fully predict that the next stimulus would be a target, and prepared the required motor response. We observed modulation of transient phase-locked ERPs, sustained ERPs, and induced oscillations, throughout this predictive sequence. These results demonstrate that predictive context optimizes target processing and speeds up target detection via the selective deployment over time of attentional and motor preparatory mechanisms activated after extraction of predictive information.

\subsection{Extraction of predictive information}

We found that the P3 amplitude increased progressively throughout the predictive sequence. This is in agreement with previous findings showing that $\mathrm{P} 3$ amplitude increases as a function of task relevance and confidence (Sawaki \& Katayama, 2006; Fogelson et al., 2009) and with a role of the P3 in context-updating (Donchin \& Coles, 1988). The P3 
amplitude enhancement with increasing predictability supports the notion that the predictive information has been extracted from the stimulus train, and that working memory has been updated accordingly. Thus, the P3 amplitude functions as a predictability index.

\subsection{Anticipation of the decisive stimulus not requiring a motor response}

Before the decisive predS3 standard stimulus, we observed a relative decrease in occipital alpha power in comparison to its non-predictive analog. As we observed in response to standard stimuli, an alpha decrease at occipital electrodes is usually observed after a visual stimulus onset, and is followed by an alpha increase (e.g. Woertz et al., 2004; Mazaheri \& Picton, 2005). These phenomena correspond to the event-related synchronization (ERS) and desynchronization (ERD) described in the alpha band (see Klimesch et al., 2007; Palva \& Palva, 2007, for reviews). This pattern of activation is thought to reflect an enhanced cortical excitability (induced by a release of inhibition) in response to the stimulus, followed by an increase in cortical inhibition after completion of stimulus processing (Klimesch et al., 2007). Several studies report that when a visual target can be anticipated, the occipital alpha power starts decreasing before stimulus onset (e.g. Worden et al., 2000; Sauseng et al., 2005; Rihs et al., 2007). The functional role of pre-stimulus occipital alpha decrease in attentional preparation has been assessed in a few studies showing, on a trial-by-trial basis, that reaction time to a predicted target decreased as a function of the pre-target alpha decrease (Thut et al., 2006; Kelly et al., 2009). In the present experiment, the occipital alpha decrease, dissociated from the need for a motor response, is felt to reflect attentiondependent preparatory activity (enhanced excitability) of the visual areas involved in processing the upcoming stimulus (predS3) that needs to be identified to fully predict a subsequent stimulus (predT). This result supports the notion that attentional preparation mechanisms, manifested by alpha decreases, can be activated in anticipation of non-target stimulus, even when no motor response is required. Noteworthy, in the present study, the pre-stimulus alpha decrease is followed by an enhancement of the post-stimulus N1 and P2 ERPs (Basar \& Stampfer, 1985; Rahn \& Basar, 1993b; a), i.e. by a facilitated sensory processing of the decisive stimulus (Hillyard et al., 1998).

Moreover, beta power decrease was found to be larger at occipital electrodes before the decisive stimulus onset, and at left central electrodes $200 \mathrm{msec}$ after stimulus onset. A beta decrease at parieto-occipital electrodes has been reported in response to visual stimuli in several studies (Mazaheri \& Picton, 2005; Deiber et al., 2007; Pesonen et al., 2007; Winterer et al., 2007; e.g. Dalal et al., 2009), but its functional role remains unknown. The left central beta decrease most likely originates in motor cortices contra-lateral to the hand pressing the button and reflects motor preparation updating. In the present work, the bilateral occipital and left central beta decreases present similar time courses, arguing for a role of the beta oscillations in visuo-motor interactions. Unfortunately, the spatial smoothing inherent to scalp EEG signals did not permit investigation of coupling in the beta band between occipital and left central electrodes.

The anticipation of a decisive stimulus, that needs to be identified but does not require a motor response, appears to mainly rely on attentional preparation mechanisms. These mechanisms operate (i) by enhancing pre-stimulus cortical excitability (alpha decrease) and facilitating post-stimulus processing (N1/P2 enhancement) in visual areas; and (ii) by increasing visuo-motor activations (beta decreases).

\subsection{Anticipation of the predicted target requiring a motor response}

A CNV response was enhanced before the predicted target, i.e. when the subject was certain about the motor response to generate. This pattern of activation indicates that the CNV is involved in several anticipatory processes (Brunia \& van Boxtel, 2001) such as motor 
preparation before the predicted target, in addition to attentional preparation. The bilateral central distribution of the $\mathrm{CNV}$ suggests a role of the $\mathrm{CNV}$ in motor planning rather than in programming the execution of the movement itself.

We observed a decrease in mu power at predicted target onset, at left central electrodes, reflecting motor cortex activation for execution of the button press (Pfurtscheller \& Lopes da Silva, 1999). Interestingly, the left central beta decrease, observed in response to all stimuli, was not larger for predicted than random targets supporting differential roles of mu and beta motor-related rhythms in motor preparation mechanisms. These results suggest that mu decrease would reflect enhanced excitability of motor areas involved in executing the upcoming movement; whereas beta reduction may be related to motor expectancy based on timing estimation and associated with visuo-motor updating. Further studies would be necessary to better decipher the role of beta and mu rhythms in motor preparation.

Noteworthy, we did not observe a pre-stimulus alpha decrease at occipital electrodes before the predicted target as it is usually observed in cue paradigms (Worden et al., 2000; Sauseng et al., 2005; Thut et al., 2006; Rihs et al., 2007). This discrepancy can be explained by the $100 \%$ predictability of the target in the present study, whereas the predictability was lower in previous research. In other words, since the subjects were sure about the identity of the upcoming target, minimal stimulus processing was required, and the visual cortices were not pre-activated. On the contrary, since the subjects were certain about the motor response to generate, motor preparation mechanisms were deployed.

Thus, anticipation of a $100 \%$ visual predictable target associated with a motor response does not require deployment of attentional preparation in the visual cortices, but seems to only rely on motor preparation mechanisms: (i) motor planning (CNV increase), followed by (ii) activation of the motor cortices involved in movement execution (mu decrease).

\subsection{Effect of anticipation mechanisms on target processing}

Target predictability and anticipation reduced target-related P2 and N2 ERPs, consistent with evidence showing that the $\mathrm{P} 2$ is involved in stimulus processing and evaluation (Potts, 2004) and that the frontal N2 is sensitive to the degree of conflict between response alternatives (Van Veen \& Carter, 2002). In particular, as observed in our data, when a target and the associated response are more probable, N2 amplitude is reduced (Gehring et al., 1992; Nieuwenhuis et al., 2003; Fogelson et al., 2010a).

Target predictability shortened P3 latency and increased P3 amplitude to targets. Shortened P3 latency to the predicted target is consistent with previous literature (Duncan-Johnson, 1981; Duncan-Johnson \& Donchin, 1982; Fogelson et al., 2009) and is ascribed to shortened duration of stimulus evaluation processing (Kutas et al., 1977; Duncan-Johnson, 1981). Our result of a larger P3 to predicted target is in contradiction with previous work reporting comparable P3 amplitudes to random and predicted targets (Fogelson et al., 2009), or showing a P3 amplitude increase with decreasing predictability (Duncan-Johnson \& Donchin, 1982; Suwazono et al., 2000) or increasing surprise (Sutton et al., 1965; Nieuwenhuis et al., 2005; Mars et al., 2008). However, our findings are in agreement with numerous studies which showed that the greater the decision confidence, the larger and earlier the P3 (Hillyard et al., 1971; Squires et al., 1975; Johnson, 1986; Picton, 1992).

Thus, predictability induces the optimization of target processing (i) by reducing stimulus evaluation and the degree of responses conflict (P2 and N2 decreases), and (ii) by enhancing decision confidence (earlier and larger P3). 


\section{Conclusions}

We demonstrated that predictive context optimizes target processing (reduced P2 and N2, earlier and larger P3) and speeds up target detection via the deployment over time of independent attention and motor preparatory mechanisms activated after extraction of predictive information. We showed that encoding of contextual cues is achieved via poststimulus sensory phase-locked ERPs and non-phase locked alpha decrease in the visual cortices; and that the extraction and implementation of the predictive information from these cues is revealed in the P3 ERP. Importantly, we observed that, during the predictive sequence, anticipation successively recruited attentional and motor preparation mechanisms by selectively pre-activating the cortical regions engaged in the processing required for the upcoming stimulus.

Prior to the occurrence of the decisive non-target stimulus to be identified, anticipation operated via attentional preparation mechanisms, indexed by enhanced excitability in visual cortices before stimulus onset (alpha decreases), and resulting in facilitated stimulus processing after stimulus onset (enhanced N1 and P2). The deployment of these mechanisms was observed even when no motor response was required.

Conversely, the subsequent $100 \%$ predictable target event showed no deployment of attentional preparation in the visual cortices. Anticipation of this fully expected target requiring a motor response operated via motor preparation mechanisms by activating motor planning mechanisms before stimulus onset (enhanced CNV), and by enhancing excitability in the motor cortices before movement onset (mu decrease).

Thus, in a predictive context, as predictability increases, anticipation operates by distinct mechanisms - namely attentional preparation and motor preparation - each dependent on what has to be accomplished with the anticipated stimulus.

\section{Supplementary Material}

Refer to Web version on PubMed Central for supplementary material.

\section{Acknowledgments}

We thank K.G. Buchanan, E. Maby and P.E. Aguera for technical support, as well as O. Bertrand, F. Barceló, U. Kraemer, B. Voytek and A. Shestyuk for helpful discussions. This work was supported by NINDS Grants NS21135 and $\mathrm{PO} 40813$.

\section{Abbreviations}

$\begin{array}{ll}\text { CNV } & \text { Contingent Negative Variation } \\ \text { ERD } & \text { Event-Related Desynchronization } \\ \text { ERP } & \text { Event-Related Potential } \\ \text { ERS } & \text { Event-Related Synchronization } \\ \text { ICA } & \text { Independent Component Analysis } \\ \text { PLF } & \text { Phase-Locking Factor } \\ \text { RT } & \text { Reaction Time } \\ \text { TF } & \text { Time-Frequency }\end{array}$




\section{References}

Aguera PE, Jerbi K, Caclin A, Bertrand O. ELAN: a software package for analysis and visualization of MEG, EEG, and LFP signals. Comput Intell Neurosci. 2011; 2011:158970. [PubMed: 21687568]

Alegre M, Gurtubay IG, Labarga A, Iriarte J, Malanda A, Artieda J. Alpha and beta oscillatory changes during stimulus-induced movement paradigms: effect of stimulus predictability. Neuroreport. 2003; 14:381-385. [PubMed: 12634488]

Basar E, Stampfer HG. Important associations among EEG-dynamics, event-related potentials, shortterm memory and learning. Int J Neurosci. 1985; 26:161-180. [PubMed: 4019045]

Blair RC, Karniski W. An alternative method for significance testing of waveform difference potentials. Psychophysiology. 1993; 30:518-524. [PubMed: 8416078]

Brunia CH, van Boxtel GJ. Wait and see. Int J Psychophysiol. 2001; 43:59-75. [PubMed: 11742685]

Dalal SS, Baillet S, Adam C, Ducorps A, Schwartz D, Jerbi K, Bertrand O, Garnero L, Martinerie J, Lachaux JP. Simultaneous MEG and intracranial EEG recordings during attentive reading. Neuroimage. 2009; 45:1289-1304. [PubMed: 19349241]

Deiber MP, Missonnier P, Bertrand O, Gold G, Fazio-Costa L, Ibanez V, Giannakopoulos P. Distinction between perceptual and attentional processing in working memory tasks: a study of phase-locked and induced oscillatory brain dynamics. J Cogn Neurosci. 2007; 19:158-172. [PubMed: 17214572]

Donchin E, Coles MGH. Is the P300 component a manifestation of context updating? The Behavioral and Brain Sciences. 1988; 11:355-425.

Duncan-Johnson CC. Young Psychophysiologist Award address, 1980. P300 latency: a new metric of information processing. Psychophysiology. 1981; 18:207-215. [PubMed: 7291436]

Duncan-Johnson CC, Donchin E. The P300 component of the event-related brain potential as an index of information processing. Biol Psychol. 1982; 14:1-52. [PubMed: 6809064]

Edgington, ES. Randomization Tests. Marcel Dekker; New York, USA: 1995.

Fogelson N, Fernandez-Del-Olmo M, Santos-Garcia D. Contextual processing deficits in Parkinson's disease: The role of the frontostriatal system. Clin Neurophysiol. 2010a

Fogelson N, Shah M, Bonnet-Brilhault F, Knight RT. Electrophysiological evidence for aging effects on local contextual processing. Cortex. 2010b

Fogelson N, Wang X, Lewis JB, Kishiyama MM, Ding M, Knight RT. Multimodal effects of local context on target detection: evidence from P3b. J Cogn Neurosci. 2009; 21:1680-1692. [PubMed: 18702574]

Gehring WJ, Gratton G, Coles MG, Donchin E. Probability effects on stimulus evaluation and response processes. J Exp Psychol Hum Percept Perform. 1992; 18:198-216. [PubMed: 1532188]

Hari R. Action-perception connection and the cortical mu rhythm. Prog Brain Res. 2006; 159:253260. [PubMed: 17071236]

Hillyard SA, Squires KC, Bauer JW, Lindsay PH. Evoked potential correlates of auditory signal detection. Science. 1971; 172:1357-1360. [PubMed: 5580218]

Hillyard SA, Vogel EK, Luck SJ. Sensory gain control (amplification) as a mechanism of selective attention: electrophysiological and neuroimaging evidence. Philos Trans R Soc Lond B Biol Sci. 1998; 353:1257-1270. [PubMed: 9770220]

Jensen O, Mazaheri A. Shaping functional architecture by oscillatory alpha activity: gating by inhibition. Front Hum Neurosci. 2010; 4:186. [PubMed: 21119777]

Jervis BW, Nichols MJ, Johnson TE, Allen E, Hudson NR. A fundamental investigation of the composition of auditory evoked potentials. IEEE Trans Biomed Eng. 1983; 30:43-50. [PubMed: 6826185]

Johnson R Jr. A triarchic model of P300 amplitude. Psychophysiology. 1986; 23:367-384. [PubMed: 3774922]

Kastner S, Pinsk MA, De Weerd P, Desimone R, Ungerleider LG. Increased activity in human visual cortex during directed attention in the absence of visual stimulation. Neuron. 1999; 22:751-761. [PubMed: 10230795] 
Kelly SP, Gomez-Ramirez M, Foxe JJ. The strength of anticipatory spatial biasing predicts target discrimination at attended locations: a high-density EEG study. Eur J Neurosci. 2009; 30:22242234. [PubMed: 19930401]

Klimesch W, Sauseng P, Hanslmayr S. EEG alpha oscillations: the inhibition-timing hypothesis. Brain Res Rev. 2007; 53:63-88. [PubMed: 16887192]

Kutas M, McCarthy G, Donchin E. Augmenting mental chronometry: the P300 as a measure of stimulus evaluation time. Science. 1977; 197:792-795. [PubMed: 887923]

Mars RB, Debener S, Gladwin TE, Harrison LM, Haggard P, Rothwell JC, Bestmann S. Trial-by-trial fluctuations in the event-related electroencephalogram reflect dynamic changes in the degree of surprise. J Neurosci. 2008; 28:12539-12545. [PubMed: 19020046]

Mazaheri A, Picton TW. EEG spectral dynamics during discrimination of auditory and visual targets. Brain Res Cogn Brain Res. 2005; 24:81-96. [PubMed: 15922161]

Nieuwenhuis S, Aston-Jones G, Cohen JD. Decision making, the P3, and the locus coeruleusnorepinephrine system. Psychol Bull. 2005; 131:510-532. [PubMed: 16060800]

Nieuwenhuis S, Yeung N, van den Wildenberg W, Ridderinkhof KR. Electrophysiological correlates of anterior cingulate function in a go/no-go task: effects of response conflict and trial type frequency. Cogn Affect Behav Neurosci. 2003; 3:17-26. [PubMed: 12822595]

Palva S, Palva JM. New vistas for alpha-frequency band oscillations. Trends Neurosci. 2007; 30:150158. [PubMed: 17307258]

Perrin F, Pernier J, Bertrand O, Echallier JF. Spherical splines for scalp potential and current density mapping. Electroencephalogr Clin Neurophysiol. 1989; 72:184-187. [PubMed: 2464490]

Perrin F, Pernier J, Bertrand O, Giard MH, Echallier JF. Mapping of scalp potentials by surface spline interpolation. Electroencephalogr Clin Neurophysiol. 1987; 66:75-81. [PubMed: 2431869]

Pesonen M, Hamalainen H, Krause CM. Brain oscillatory 4-30 Hz responses during a visual n-back memory task with varying memory load. Brain Res. 2007; 1138:171-177. [PubMed: 17270151]

Pfurtscheller G, Lopes da Silva FH. Event-related EEG/MEG synchronization and desynchronization: basic principles. Clin Neurophysiol. 1999; 110:1842-1857. [PubMed: 10576479]

Picton TW. The P300 wave of the human event-related potential. J Clin Neurophysiol. 1992; 9:456479. [PubMed: 1464675]

Posner MI. Orienting of attention. Q J Exp Psychol. 1980; 32:3-25. [PubMed: 7367577]

Potts GF. An ERP index of task relevance evaluation of visual stimuli. Brain Cogn. 2004; 56:5-13. [PubMed: 15380870]

Rahn E, Basar E. Enhancement of visual evoked potentials by stimulation during low prestimulus EEG stages. Int J Neurosci. 1993a; 72:123-136. [PubMed: 8225796]

Rahn E, Basar E. Prestimulus EEG-activity strongly influences the auditory evoked vertex response: a new method for selective averaging. Int J Neurosci. 1993b; 69:207-220. [PubMed: 8083007]

Rihs TA, Michel CM, Thut G. Mechanisms of selective inhibition in visual spatial attention are indexed by alpha-band EEG synchronization. Eur J Neurosci. 2007; 25:603-610. [PubMed: 17284203]

Sauseng P, Klimesch W, Stadler W, Schabus M, Doppelmayr M, Hanslmayr S, Gruber WR, Birbaumer N. A shift of visual spatial attention is selectively associated with human EEG alpha activity. Eur J Neurosci. 2005; 22:2917-2926. [PubMed: 16324126]

Sawaki R, Katayama J. Stimulus context determines whether non-target stimuli are processed as taskrelevant or distractor information. Clin Neurophysiol. 2006; 117:2532-2539. [PubMed: 17005448]

Squires KC, Squires NK, Hillyard SA. Decision-related cortical potentials during an auditory signal detection task with cued observation intervals. J Exp Psychol Hum Percept Perform. 1975; 1:268279. [PubMed: 1202150]

Sutton S, Braren M, Zubin J, John ER. Evoked-potential correlates of stimulus uncertainty. Science. 1965; 150:1187-1188. [PubMed: 5852977]

Suwazono S, Machado L, Knight RT. Predictive value of novel stimuli modifies visual event-related potentials and behavior. Clin Neurophysiol. 2000; 111:29-39. [PubMed: 10656508]

Tallon-Baudry C, Bertrand O. Oscillatory gamma activity in humans and its role in object representation. Trends Cogni Sci. 1999; 3:151-162. 
Tallon-Baudry C, Bertrand O, Delpuech C, Pernier J. Stimulus specificity of phase-locked and nonphase-locked $40 \mathrm{~Hz}$ visual responses in human. J Neurosci. 1996; 16:4240-4249. [PubMed: 8753885]

Thut G, Nietzel A, Brandt SA, Pascual-Leone A. Alpha-band electroencephalographic activity over occipital cortex indexes visuospatial attention bias and predicts visual target detection. $\mathrm{J}$ Neurosci. 2006; 26:9494-9502. [PubMed: 16971533]

Trenner MU, Heekeren HR, Bauer M, Rossner K, Wenzel R, Villringer A, Fahle M. What happens in between? Human oscillatory brain activity related to crossmodal spatial cueing. PLoS One. 2008; 3:e1467. [PubMed: 18213376]

Van Veen V, Carter CS. The timing of action-monitoring processes in the anterior cingulate cortex. J Cogn Neurosci. 2002; 14:593-602. [PubMed: 12126500]

Voisin J, Bidet-Caulet A, Bertrand O, Fonlupt P. Listening in silence activates auditory areas: a functional magnetic resonance imaging study. J Neurosci. 2006; 26:273-278. [PubMed: 16399697]

Winterer G, Carver FW, Musso F, Mattay V, Weinberger DR, Coppola R. Complex relationship between BOLD signal and synchronization/desynchronization of human brain MEG oscillations. Hum Brain Mapp. 2007; 28:805-816. [PubMed: 17133396]

Woertz M, Pfurtscheller G, Klimesch W. Alpha power dependent light stimulation: dynamics of eventrelated (de)synchronization in human electroencephalogram. Brain Res Cogn Brain Res. 2004; 20:256-260. [PubMed: 15183396]

Worden MS, Foxe JJ, Wang N, Simpson GV. Anticipatory biasing of visuospatial attention indexed by retinotopically specific alpha-band electroencephalography increases over occipital cortex. J Neurosci. 2000; 20:RC63. [PubMed: 10704517] 


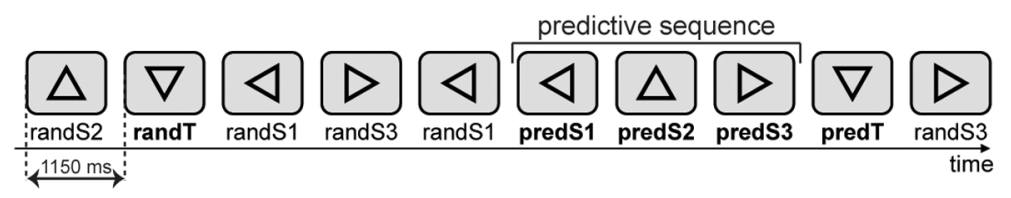

Fig. 1. Stimuli

A sequence of triangles was centrally presented on a screen. Stimuli consisted of $15 \%$ targets (downward-facing triangle) and $85 \%$ of equal amounts of three types of standard stimuli: triangles facing upward (randS2), left (randS1), and right (randS3). A random sequence of standards preceded non-predictable random targets (randT); whereas a predictive sequence of three standards facing left, upward and right always preceded predictable targets (predT). Triangles of the predictive sequence are labeled as predS1, predS2 and predS3 stimuli. predS3 is the decisive stimulus $100 \%$ predicting a subsequent target. 

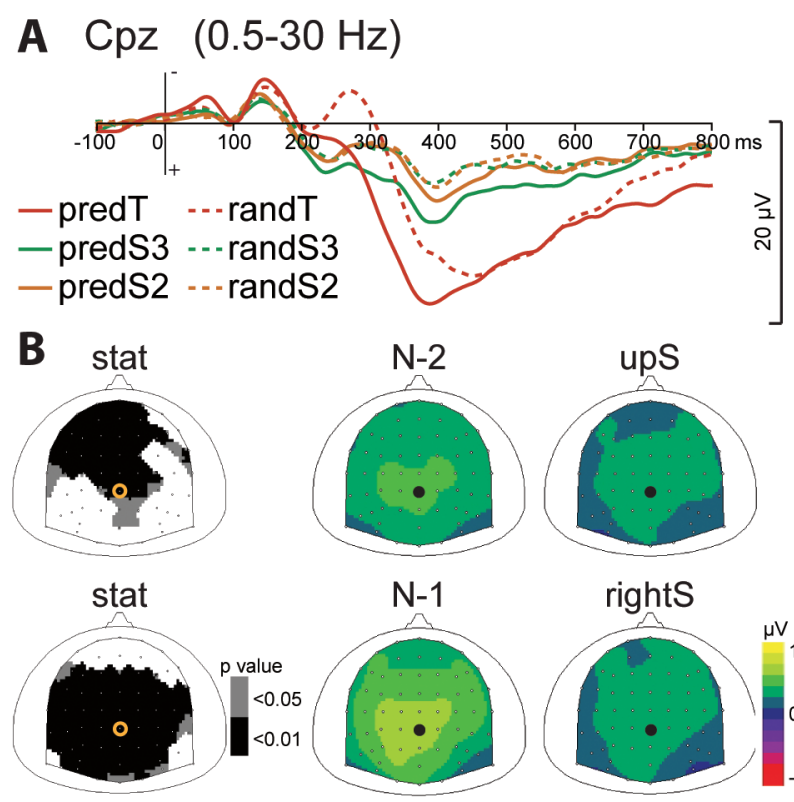

$\mathrm{N}-1$
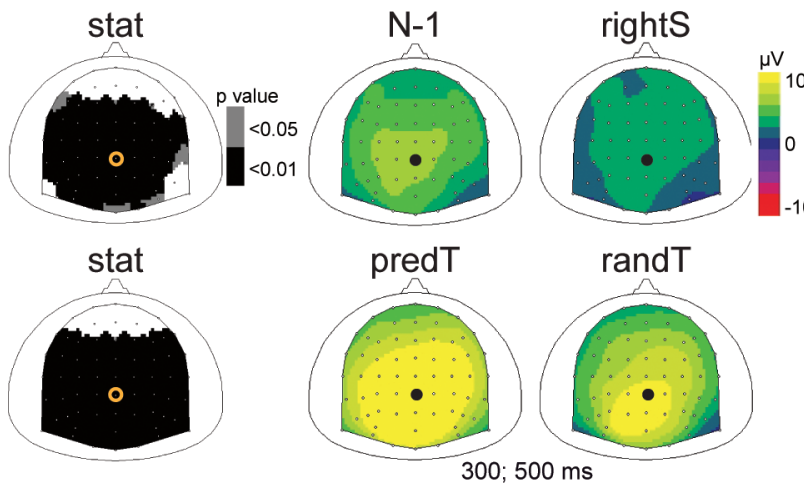

Fig. 2. Effect of predictive context on the P3 ERP

(A) Grand-average ERP waveforms band-pass filtered between .5 and $30 \mathrm{~Hz}$, at $\mathrm{Cpz}$ electrode. ERPs to predictive stimuli (predS2, predS3 and randT) and their non-predictive analogs (randS3, randS2 and randT) are depicted with full and dashed lines, respectively.

(B) Scalp topographies (top views) of the P3 ERP for each pair of predictive stimulus and its non-predictive analog. Left column: topographies of the p-value resulting from permutation tests at the latency of the maximal difference $(435 \mathrm{msec}$ for predS2/randS2, 390msec for predS3/randS3, and 310msec for predT/randT). Right columns: topographies of the mean ERP values in the 300 to $500 \mathrm{msec}$ time-window. The black dots and yellow circles indicate the position of the Cpz electrode. 
A $\quad \mathrm{PO} 4 \quad(4-30 \mathrm{~Hz})$

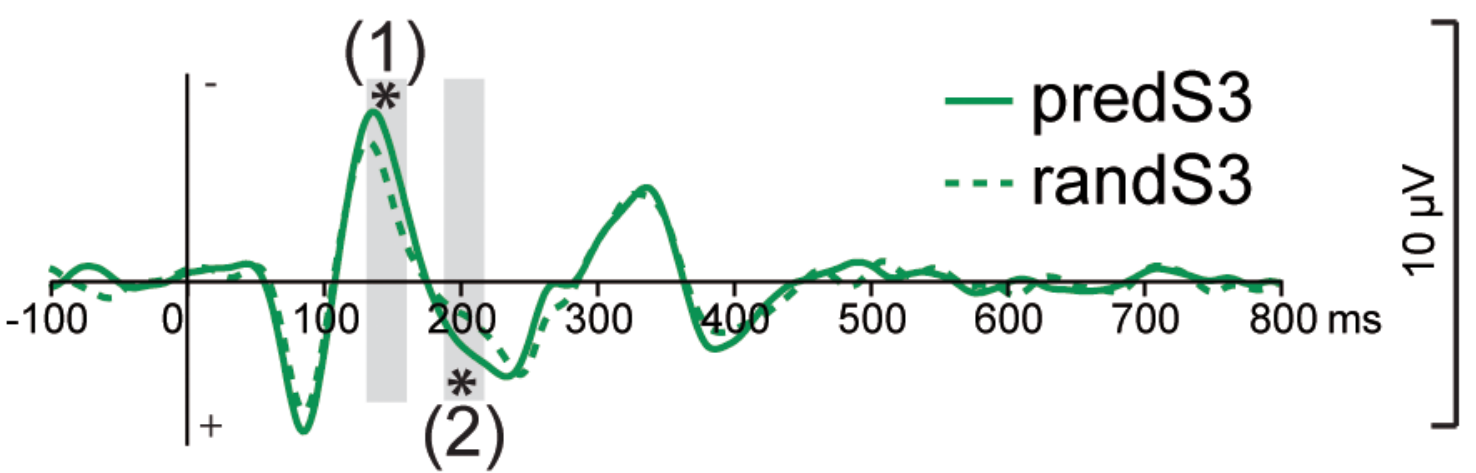

B

stat

predS3

randS3
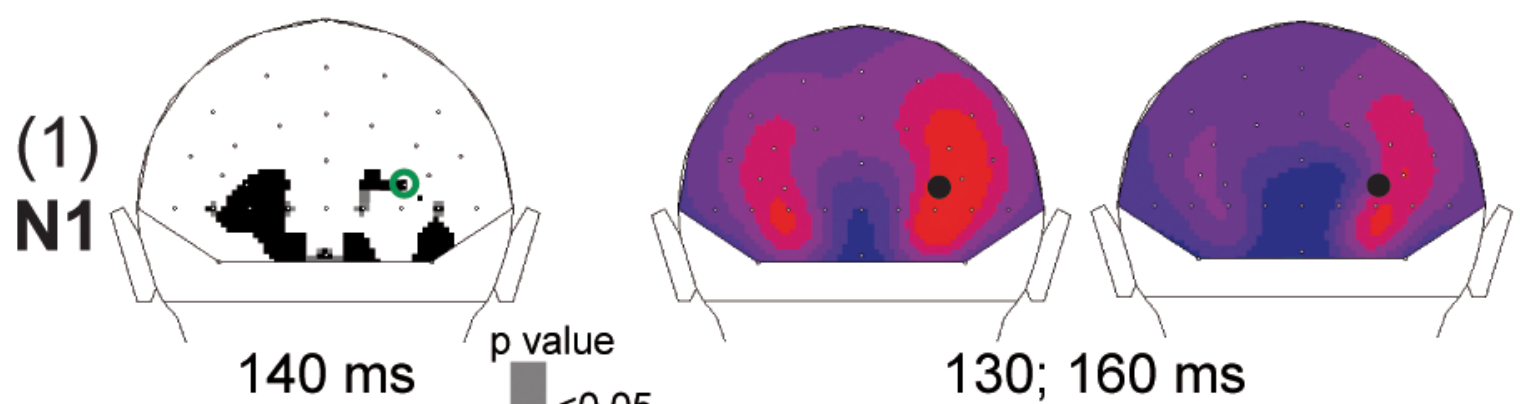

$\mu \mathrm{V}$
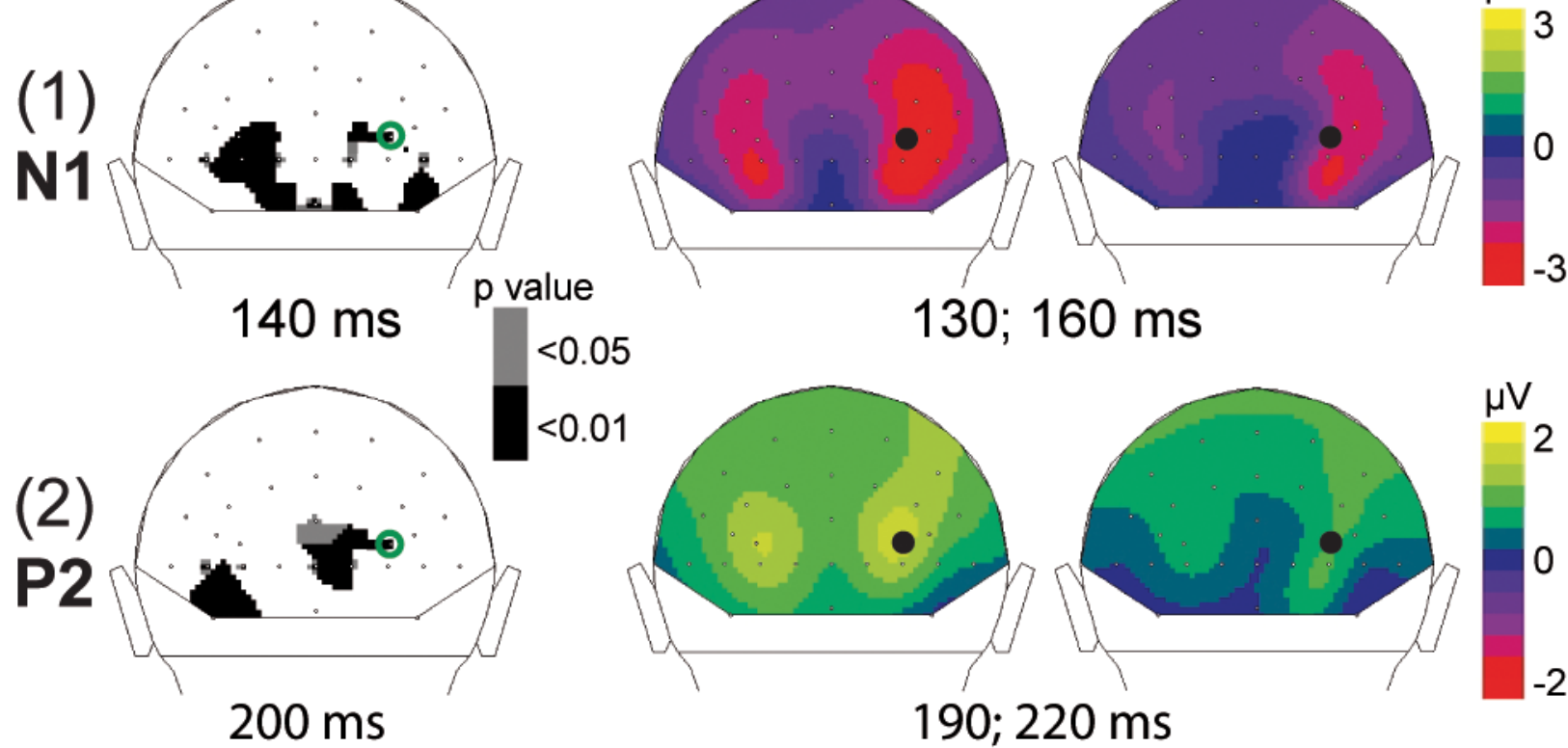

Fig. 3. Effect of predictive context on early visual ERPs

(A) Grand-average ERP waveforms band-pass filtered between 4 and $30 \mathrm{~Hz}$, at PO4 electrode. ERPs to the decisive predS3 stimulus and its non-predictive analog (randS3) are depicted with full and dashed green lines, respectively. Time-windows showing significant difference between the 2 conditions are indicated by grey bars $(*: \mathrm{p}<.01)$. (B) Scalp topographies (back views) of the early N1 (1) and P2 (2) ERPs. Left column: topographies of the p-value resulting from permutation tests at the latency of the maximal difference (140msec for N1, 200msec for P2). Right columns: topographies of the mean ERP values in the time-windows indicated by grey bars in (A). The black dots and green circles indicate the position of the $\mathrm{PO} 4$ electrode. 


\section{A Cz $(0.5-30 \mathrm{~Hz})$}
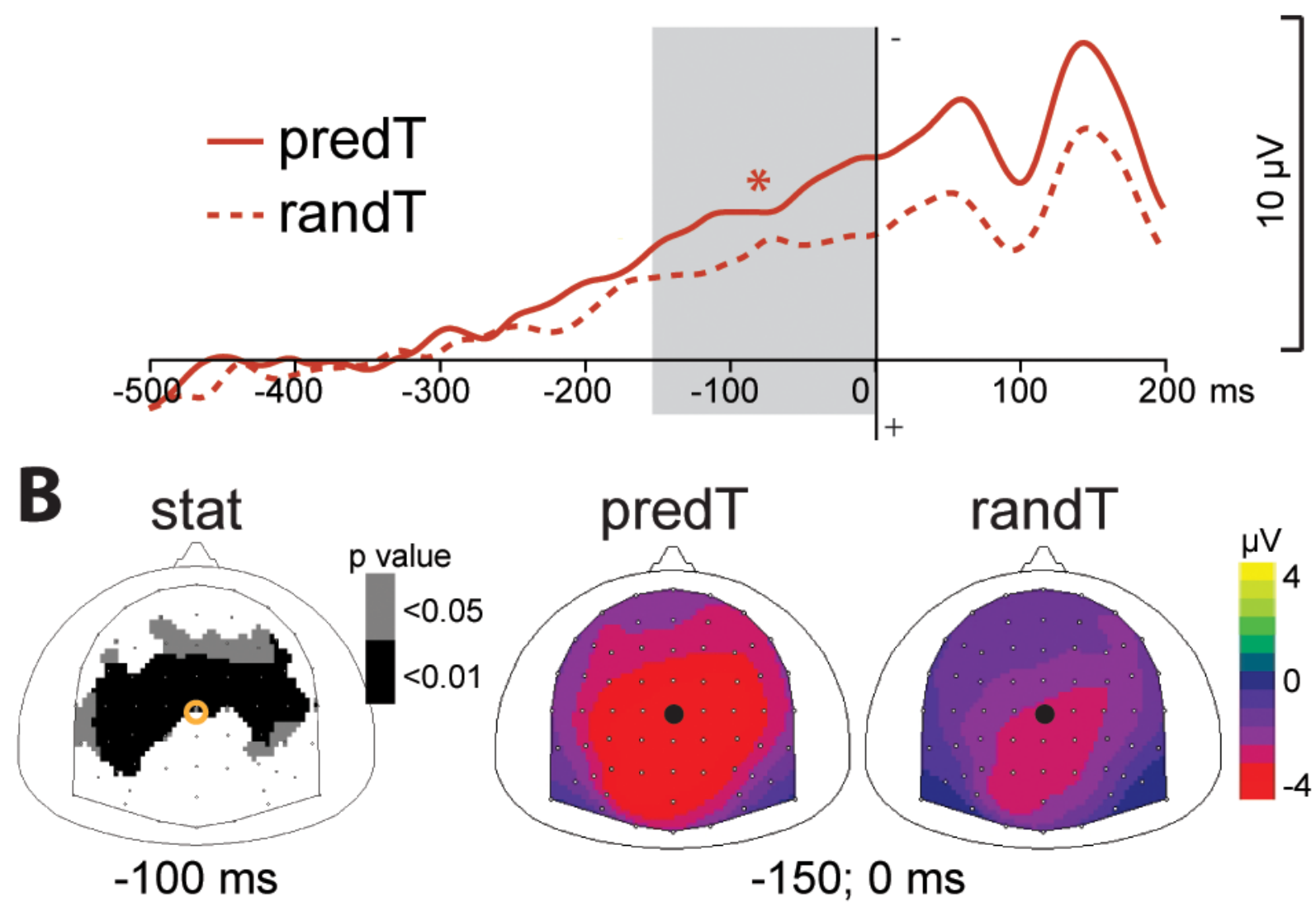

Fig. 4. Effect of predictive context on the CNV ERP

(A) Grand-average non baseline corrected ERP waveforms band-pass filtered between 0.5 and $30 \mathrm{~Hz}$, at $\mathrm{Cz}$ electrode. ERPs to the predicted (predT) and random (randT) targets are depicted with full and dashed red lines, respectively. The time-window showing a significant difference between predT and randT conditions is indicated by a grey bar $(*: \mathrm{p}<$. 01). (B) Scalp topographies (top views) of the CNV ERP in response to predT and randT. Left column: topographies of the p-value resulting from permutation tests at $-100 \mathrm{~ms}$. Right columns: topographies of the mean ERP values in the time-window indicated by the grey bar in (A), i.e. between -150 and $0 \mathrm{~ms}$. The black dots and yellow circles indicate the position of the $\mathrm{Cz}$ electrode. 
A $\mathrm{FCz}(4-30 \mathrm{~Hz})$

(2)

- predT

- - - randT

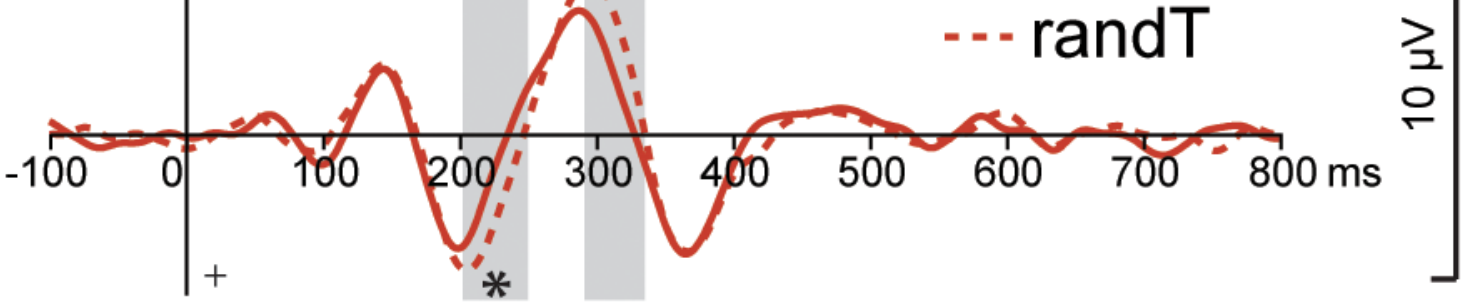

(1)

B

stat
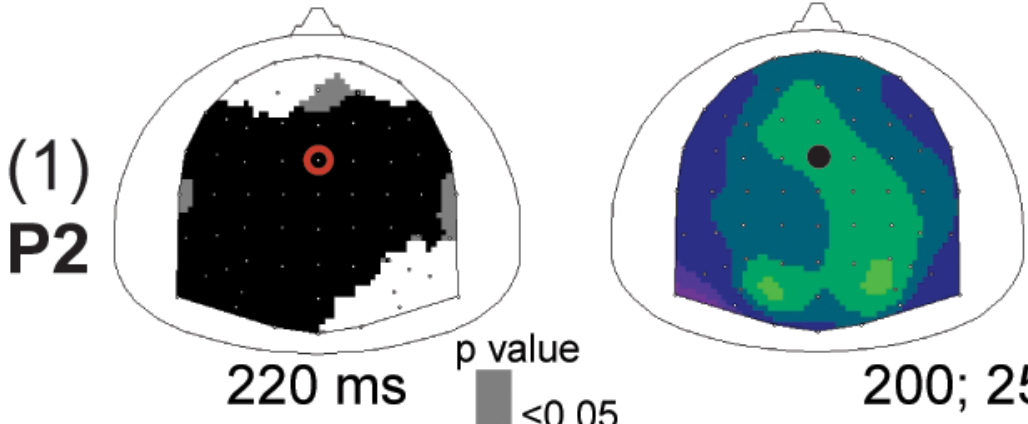

predT

randT
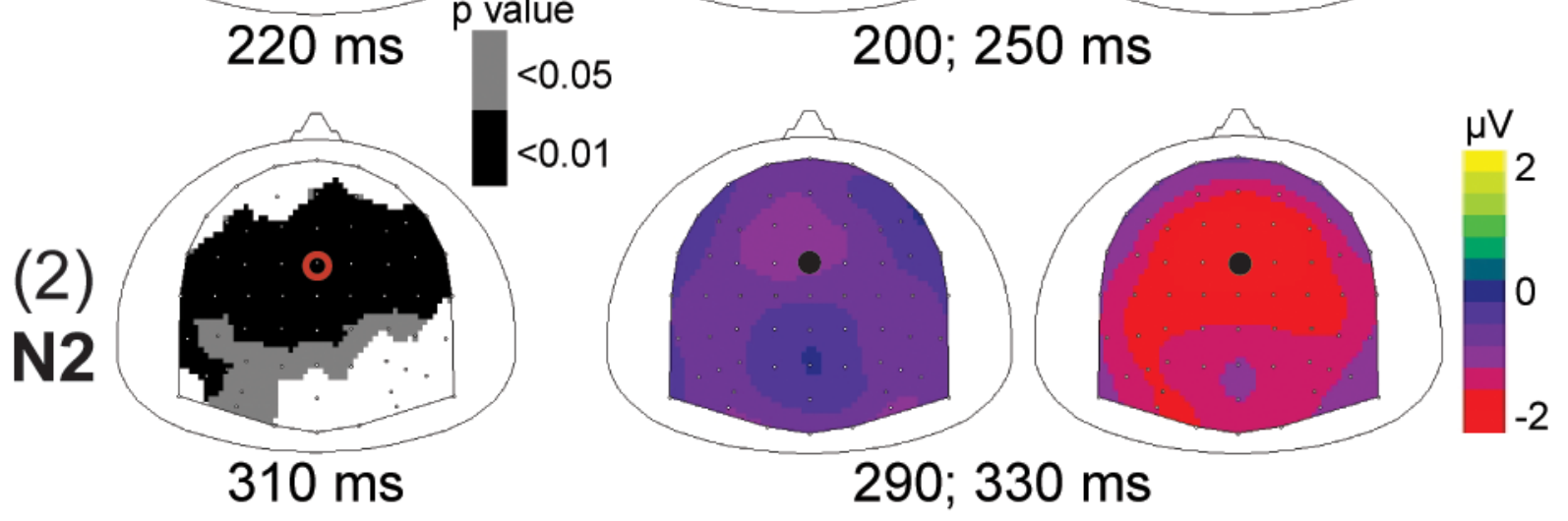

Fig. 5. Effect of predictive context on early ERPs to targets

(A) Grand-average ERP waveforms band-pass filtered between 4 and $30 \mathrm{~Hz}$, at $\mathrm{FCz}$ electrode. ERPs to the predicted (predT) and random (randT) targets are depicted with full and dashed red lines, respectively. Time-windows showing significant difference between the 2 conditions are indicated by grey bars $(*: p<.01)$. (B) Scalp topographies (top views) of the early P2 (1) and N2 (2) ERPs. Left column: topographies of the p-value resulting from permutation tests at the latency of the maximal difference (220ms for P2, 310msec for N2). Right columns: topographies of the mean ERP values in the time-windows indicated by grey bars in (A). The black dots and red circles indicate the position of the FCz electrode. 

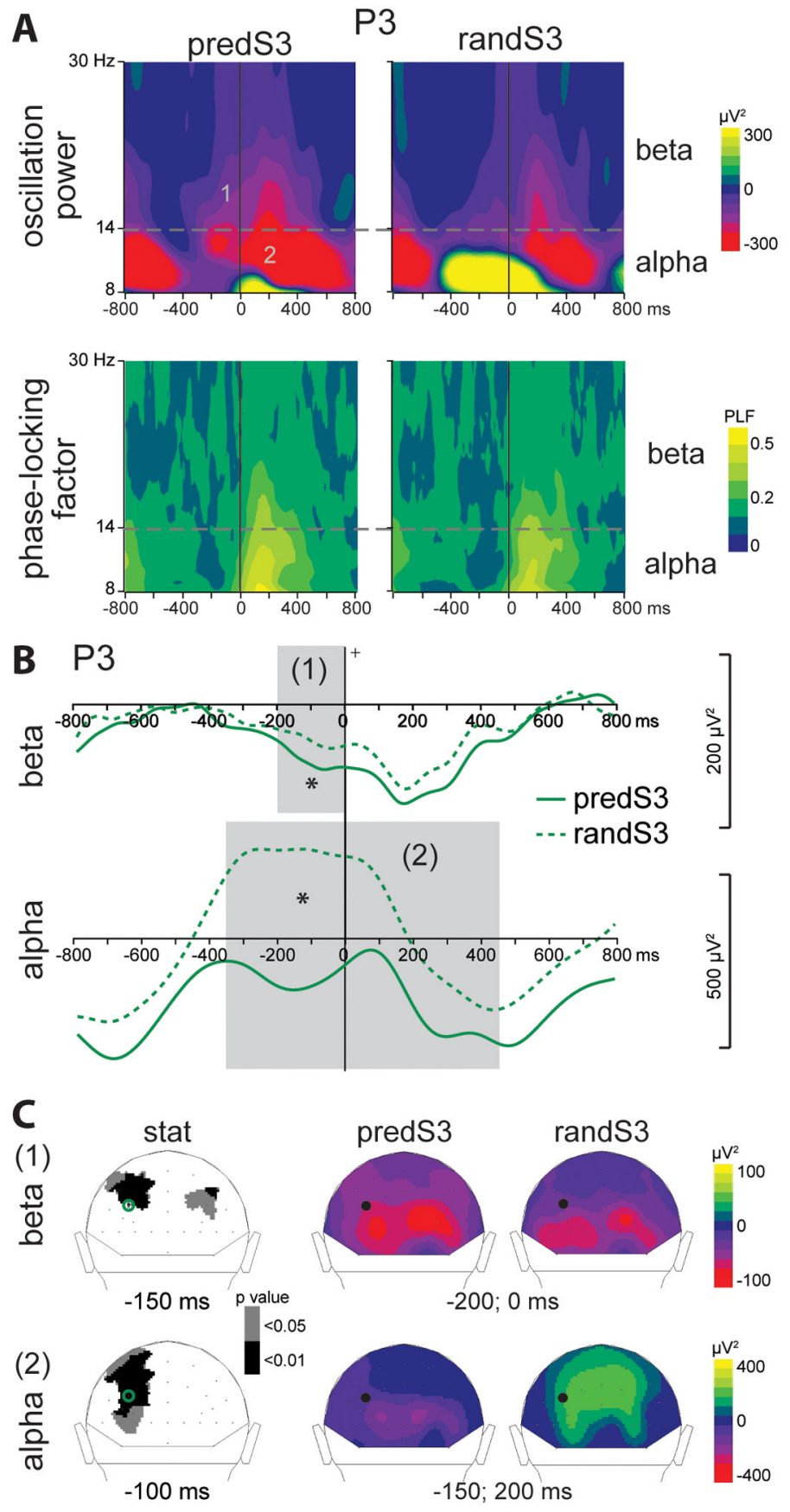

Fig. 6. Effect of predictive context on oscillatory activities

(A) Grand-average time-frequency (TF) plots of the oscillation power (upper panel) and of the phase-locking factor (lower panel) at $\mathrm{P} 3$ electrode, in response to the decisive predS3 stimulus and its non-predictive analog (randS3). Alpha $(8-14 \mathrm{~Hz})$ and beta $(14-30 \mathrm{~Hz})$ frequency bands are depicted.

(B) Beta and alpha frequency band profiles of TF power at P3 electrode. Profiles to randS3 and predS3 stimuli are depicted with full and dashed green lines respectively. Timewindows showing significant difference between the 2 conditions are indicated by grey bars (*: p <.01). (C) Left column: scalp topographies (back views) of the p-value resulting from 
permutation tests in the alpha and beta frequency ranges. Right columns: scalp topographies of the mean TF power value in the indicated time-windows. The black dots and green circles indicate the position of the $\mathrm{P} 3$ electrode. In response to the decisive predS3 stimulus, a prestimulus larger beta decrease (1) comes with a smaller alpha increase (2), at parieto-occipital electrodes. 

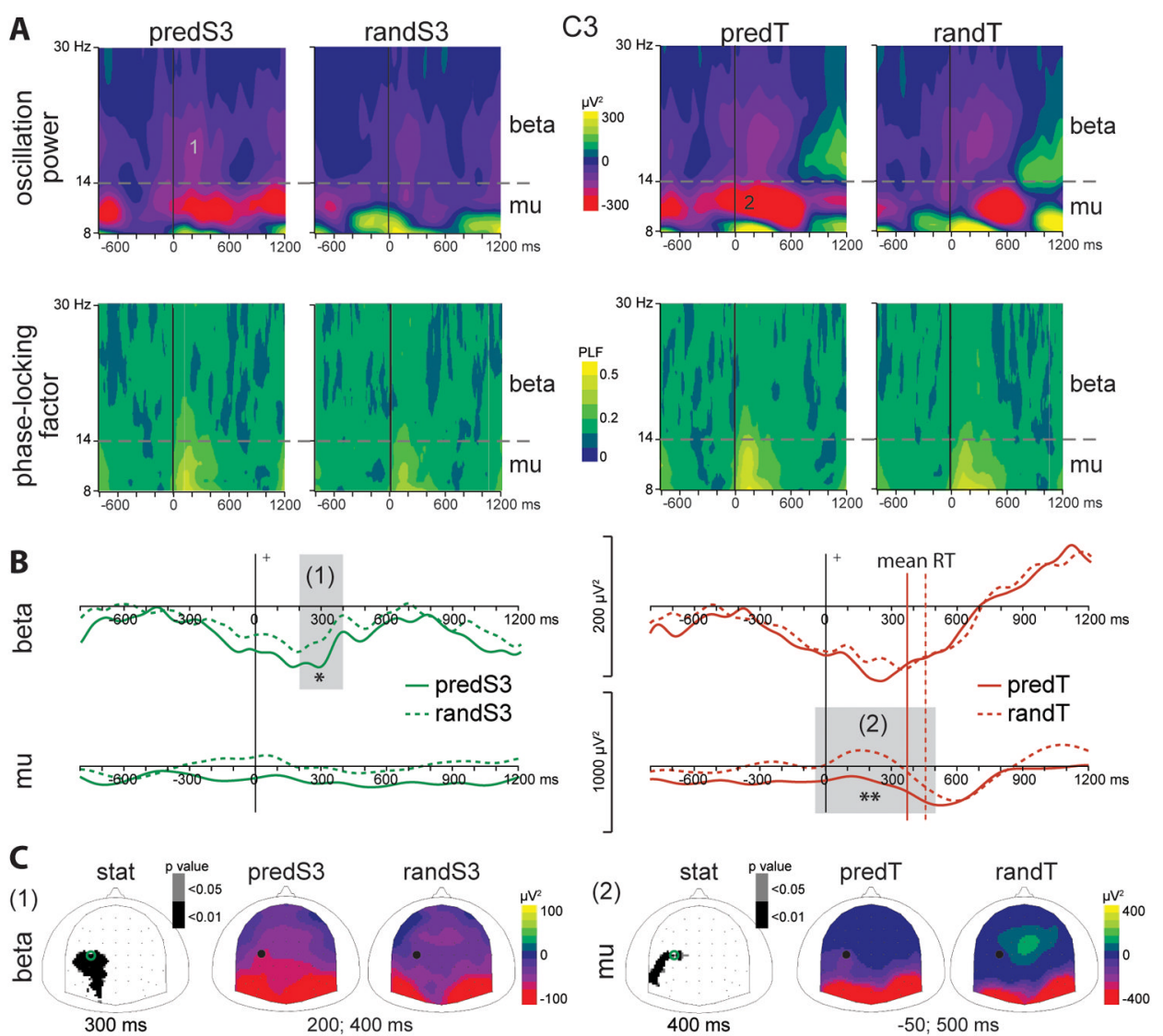

Fig. 7. Effect of predictive context on motor oscillatory activities

Left panel: comparison of predS3 and randS3 stimuli. Right panel: comparison of predT and randT stimuli. (A) Grand-average time-frequency (TF) plots of the oscillation power (upper panel) and of the phase-locking factor (lower panel) at C3 electrode. $\mathrm{Mu}(8-14 \mathrm{~Hz})$ and beta $(14-30 \mathrm{~Hz})$ frequency bands are depicted. (B) Beta and mu frequency band profiles of TF power at $\mathrm{C} 3$ electrode. Profiles to predS3 and randS3 stimuli are depicted with full and dashed green lines, respectively. Profiles to predT and randT stimuli are depicted with full and dashed red lines, respectively. The mean reaction time (RT) to predT or randT is indicated by a vertical full or dashed red line, respectively. Time-windows showing significant difference between the 2 conditions are indicated by grey bars $(*: \mathrm{p}<.01 ; * *: \mathrm{p}$ $<.001$ ). (C) Left column: scalp topographies (top views) of the p-value resulting from permutation tests in the beta (1) and mu (2) frequency ranges. Right columns: scalp topographies of the mean TF power value in the time-windows indicated by grey bars in (B) and labeled in (A). The dots and circles indicate the position of the $\mathrm{C} 3$ electrode. 